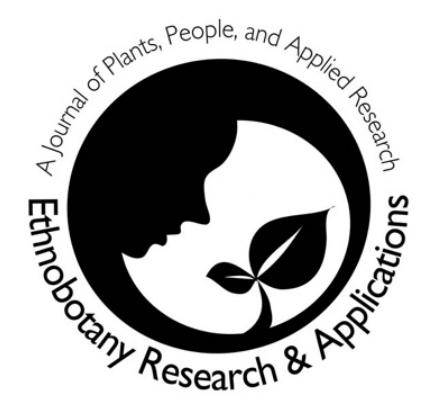

\title{
Medicinal plant species used in the treatment of skin diseases in Katabi Sub- County, Wakiso District, Uganda
}

\author{
Cissy Nambejja, Patience Tugume, Clement Nyakoojo, \\ Maud Kamatenesi-Mugisha
}

\section{Research}

\begin{abstract}
Background: The use of medicinal plants in primary healthcare is central among rural communities in Uganda due to the expensive and often inaccessible conventional healthcare. Skin diseases pose a serious health risk given the challenge of HIV-AIDS whose opportunist infections manifest themselves as skin complications. The study investigated, identified and documented plant species used to manage skin infections, the plant parts used, methods of preparation and administration of herbal remedies.
\end{abstract}

Methods: An ethnobotanical survey of medicinal plant species used to treat skin diseases was conducted in Katabi Sub-County, Wakiso District in Uganda using semi structured interviews. A questionnaire, checklist, and personal observation were the research instruments used. Descriptive statistics were used to present the data.

Results: A total of 60 respondents were interviewed from 20 villages during this study. 50 plant species belonging to 29 families were documented. The highest proportion of species $(25 \%)$ was used against fungal infections. Other proportions of plant species against their respective diseases were; $24 \%$, $20 \%, 16 \%$ and $16 \%$ for allergies, wounds, skin rash and warts respectively and were inclusive of each other. Other mentioned diseases were treated by low proportions of plant species. The most frequently used plants were Psorospermum febrifugum (33\%), Albizia coriaria (32\%), Hoslundia opposita (37\%), Justicia betonica (30\%); and Euphorbia tirucalli (25\%) Herbs (55\%) were the main growth habit of medicinal plant species followed by trees $(25 \%)$, shrubs (17\%) and vines (2\%) and grasses (1) Leaves $(64 \%)$ were the most commonly used plant parts, followed by bark and roots ( $14 \%$ each), fruits $(10 \%)$, flowers $(8 \%)$, sap $(5 \%)$ and pseudostem $(2 \%)$. Fungal infections had the highest informant consensus factor (0.9) and Justicia betonica had the highest fidelity level (89\%) for treatment of fungal infections. Topical administration to affected parts was the major method of administration at $54 \%$. Other methods were oral, bathing, compresses, poultices and inhalation.

\section{Correspondence}

Cissy Nambejja ${ }^{1}$, Patience Tugume ${ }^{2^{*}}$, Clement Nyakoojo $^{2}$, Maud Kamatenesi-Mugisha ${ }^{2}$

${ }^{1}$ Natural Chemotherapeutics Research Institute, P.O Box 7272, Kampala, Uganda

${ }^{2}$ Department of Plant Sciences, Microbiology \& Biotechnology, Makerere University, P.O Box 7062, Kampala, Uganda, B.P: 11201 Meknes,

*Corresponding author: ptugume@gmail.com

Ethnobotany Research \& Applications 18:20 (2019)

Conclusion: The traditional knowledge about the use of medicinal plants for management of skin diseases was diverse in Katabi Sub-County. Fungal infections were the most common skin diseases. The dominant plant species used were; Psorospermum febrifugum, Albizia coriaria, Hoslundia opposita, Justicia betonica, and Euphorbia tirucalli. 
Keywords: Skin diseases, Wakiso, medicinal plants, Uganda, topical administration.

\section{Background}

Worldwide use of medicinal plants for primary healthcare is on the increase (Karuiki \& Njoroge 2011). The World Health Organization (WHO 2002) estimated that about $80 \%$ of the people in developing countries depend on local medicinal plants for primary health care.

The greater part of the traditional therapy involves the use of plant for treatment of various diseases. Many cultures have a deep understanding of properties of plants, knowledge that is endangered due to lack of documentation. Plants parts, have clear pharmacological activity in our bodies, ranging from very subtle to profound (Gollin 2004). Plant based pharmaceuticals are considered worth pursuing because there is increasing awareness of the loss of biodiversity throughout the world, a loss that may preclude future discoveries of plant- based pharmaceuticals (Chen et al. 2016). In a bid to preserve traditional medicine knowledge, there is need to carry out inventories of plants with therapeutic value.

Healthcare practices of a community are dependent on many factors which may be physical, socioeconomical, cultural or political (Kroeger 1983) and the healthcare system in existence (Shaikh \& Hatcher 2005). In Katabi Sub-County, most households are in the low income bracket which prohibits them from utilization of modern health services and resort to traditional medicine (Musoke et al. 2014). Most residents of the area prefer selfmedication using local herbs or soliciting the services of traditional healers (Musoke et al. 2014). Rural people believe that traditional medicine has minimal side effects on the human body as opposed to many drugs in western medical practices (Galabuzi et al 2010).

The widespread use of medicinal plant species in rural Uganda is attributed to inaccessibility to modern health facilities, poverty, socio-cultural beliefs, inadequate medicines and other supplies, shortage and low motivation of the modern medical staff and the fact that traditional medicine has a wide acceptance. (Kamatenesi et al. 2011). Socio-cultural factors associated with the demand for traditional medicine include; beliefs and perception of illness, cultural norms, taboos, religious affiliations and household economic status (Galabuzi et al. 2010). A patient who believes the cause of a disease is of spiritual origin will seek care from spiritual healers even when the disease is not associated with spirits or curses. However, a saved christian may rely on prayers as a form of treatment. In addition, there is growing resistance of various disease causing agents to allopathic medicine necessitating discovery of new drugs by conducting ethnobotanical studies (Mahbub et al. 2018). The use of medicinal plant species varies according to nature of the disease (Vikram et al. 2011). For skin infections most remedies are applied topically but at times herbs are mixed with each other or other products like oil and clay before application (Iwu 1993). In some cases plant decoctions are used directly on the wound or infected part.

Skin conditions are the abnormal or unusual appearances on the skins of adults and children in form of blisters, ulcers or rashes (Tabassum \& Hamdani 2014). Skin conditions are a very common problem in Ugandan communities due to rapid urbanization that results in overcrowding in slums (Ahumuza \& Kirimuhuzya 2011). Provision of treatment for these infections constrains household budgets so most families resort to the use of herbal medicine as a cheaper option.

Skin infections are common among pupils because of the lifestyles they lead especially in boarding schools where combs, razor blades, basins, sweaters and clothes are shared (Morakinyo et al. 2014). This exposes them to a wide range of fungal skin infections. In Uganda most local saloons cannot afford chemicals and equipment for sterilization of the tools used when cutting hair leading to rampant transmission of skin infections mainly of the head and neck. Such tools contain viable fungal spores which are transmitted from one person to another through micro-traumas and skin macerations during hair cutting (Uslu et al. 2008).

Traditional knowledge on treatment of various diseases is passed on from one generation to the next without any documentation (Badoni \& Badoni 2001, Kala 2007), yet most holders of such knowledge are the elderly who may die before passing it on to the young generation. It is against this background that medicinal plant species used to manage skin infections and associated indigenous knowledge in Katabi Sub-County, Wakiso District were documented. This will ensure that traditional knowledge about use of plant species in management of skin infections is conserved. It will also form a basis for discovery of new drugs.

\section{Materials and Methods}

\section{Study Area}

Katabi Sub-County is located in Wakiso District, Central Uganda between $0^{0} 50^{\prime} 0^{\prime \prime} \mathrm{N}$ and $32^{\circ} 28^{\prime} 0^{\prime \prime} \mathrm{E}$. Katabi Sub-County comprises five parishes namely Kabale, Kisubi, Kitala, Nalugala, and Nkumba. There is one hospital in the area called Kisubi .The Katabi Community has a close proximity to Entebbe- 
Kampala Road and contains isolated fishing villages that extend into the marshland of Lake Victoria. Luganda is the main language spoken in the area. The people of Katabi are mainly subsistence farmers of maize, bananas, cassava, sweet potatoes, beans and yams.

\section{Sampling Design}

Key informants were interviewed. The survey covered all the five parishes of the sub-county. Interviewees from four villages of each parish were randomly selected. Hebron Junior School in Nalugala Parish was visited and pupils observed for prevalence of skin infections. Household data were collected using a questionnaire that comprised open ended questions. In-depth discussions guided by a checklist were held with key informants who are knowledgeable about the use of medicinal plant species and skin infections.

Data were collected about the common skin diseases, medicinal plant species used to manage them, plant parts used, mode of preparation and administration. With the help of traditional healers as guides, all medicinal plant species mentioned were collected and identified in the field based on a manual for plant identification (Katende et al. 1999). Plants that could not be identified were collected and identified at the Makerere University Herbarium. Correctness of scientific names of species was checked according to Tropicos:http://www.tropicos.org database accessed on $31^{\text {st }}$ January, 2019. The data were presented using descriptive statistics. The skin diseases were put into different categories. Informant consensus factor $\left(F_{i c}\right)$ for the dominantly used species was calculated to indicate the homogeneity of information using the following formula proposed by (Trotter \& Logan 1986);

$F_{\text {ic }}=\frac{\text { Nur-Ntaxa }}{\text { Nur-1 }}$

Where Nur $=$ Number of use reports

Ntaxa $=$ Number of species in each use category

The Fidelity levels for the dominantly used plant species (FL) were calculated according to Friedman et al. (1986).

$\mathrm{FL}=\mathrm{I}_{\mathrm{p}} / \mathrm{I}_{\mathrm{u}} \times 100$

Where $I_{p}=$ Number of informants who suggested the use of a species for the same major skin condition
$\mathrm{I}_{\mathrm{u}}=$ the total number of informants who mentioned the species for any use.

\section{Results and Discussion}

Characteristics of respondents

A total of 60 respondents who included a medical doctor and two nurses were interviewed out of which $81 \%$ were females and $19 \%$ males. The majority of the respondents $(52 \%)$ were aged between $31-45$ years. The high number of females could be attributed to the fact that women are usually caretakers of children who are prone to skin infections and thus often search for remedies of skin infections. This corroborates findings of other ethnobotanical studies in Uganda (Tugume et al. 2016) and Rwanda (Kamagaju et al. 2013). The distribution of respondents by age group and gender is shown in Fig. 1.

Individuals above 60 years possessed more knowledge compared to those below 60 . On average each respondent aged 60 years and above mentioned 20 plants while those below 60 years named on average five plant species. This calls for the need to document traditional medicine practices to avoid indigenous knowledge erosion in case the elderly die before transmission of such knowledge. This loss will also be exacerbated by the extinction of plant species due to urban expansion and habitat destruction. Diversity of medicinal plant species used to manage skin diseases

A total of 50 plant species belonging to 29 families were documented (Table 1). Asteraceae had the highest number of plant species (seven) harvested followed by Euphorbiaceae and Fabaceae (five each). $36 \%$ of plant species were used to treat fungal infections (Table 2). Families Solanaceae and Lamiaceae had three species each; Acanthaceae, Brassicaceae and Curcurbitaceae had two species each while Alliaceae, Aloeaceae, Amaranthaceae, Apiaceae, Bignoniaceae, Burseraceae, Capparaceae, Caricaceae, Clusiaceae, Dracaenaceae, Lauraceae, Moraceae, Moringaceae, Musaceae, Myrtaceae, Phyllanthaceae, Phytolacaceae, Piperaceae, Poaceae and Zingeberaceae had one species each. Dominance of these families as sources of medicine has been reported by other ethnobotanical studies (Lulekal et al. 2008, Namukobe et al. 2011). This could be attributed to their widespread distribution and abundance (Wondimu et al. 2007) and rich bioactive ingredients (Thomas et al. 2009). 


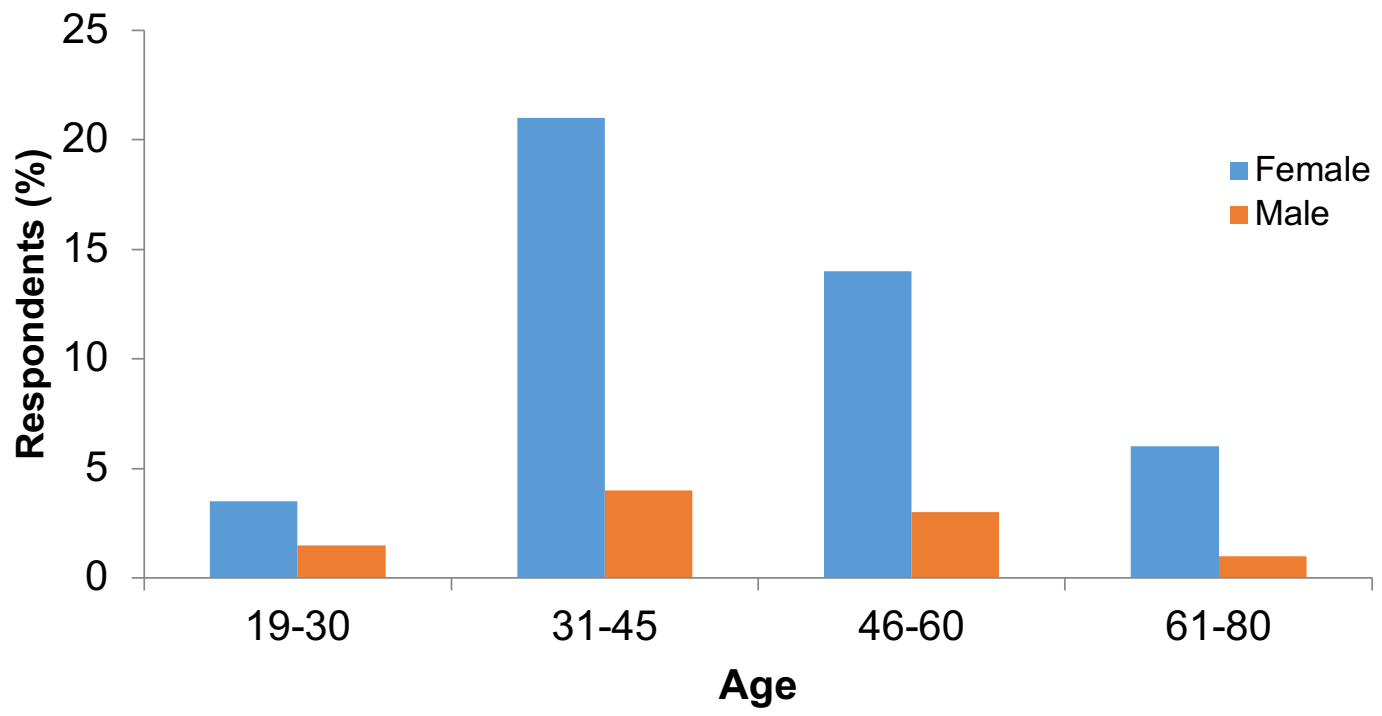

Flg. 1 Gender and age distribution of respondents

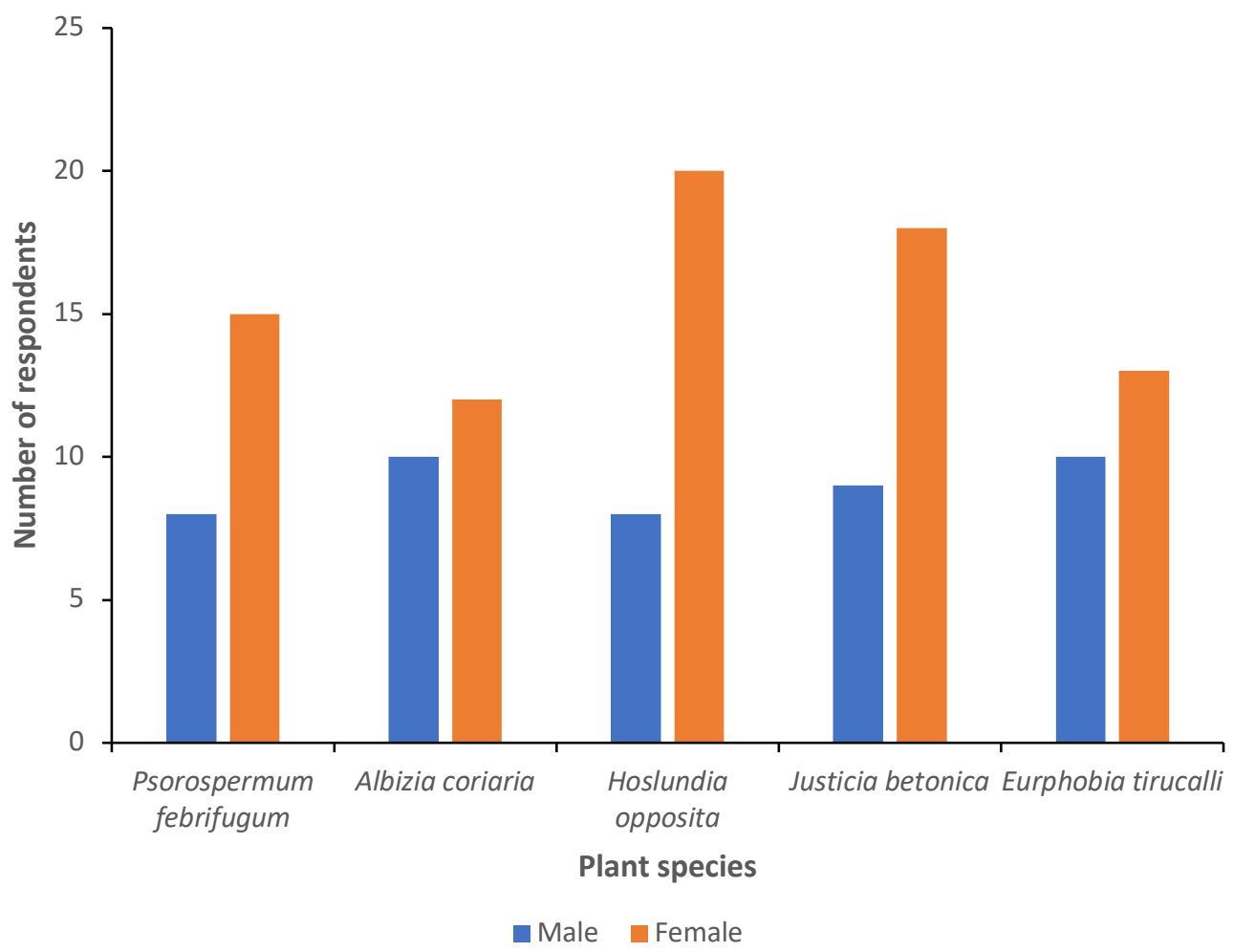

Fig. 2. Dominant species according to number of respondents and gender 
Table 1. The Medicinal Plant species used to Treat Skin Diseases

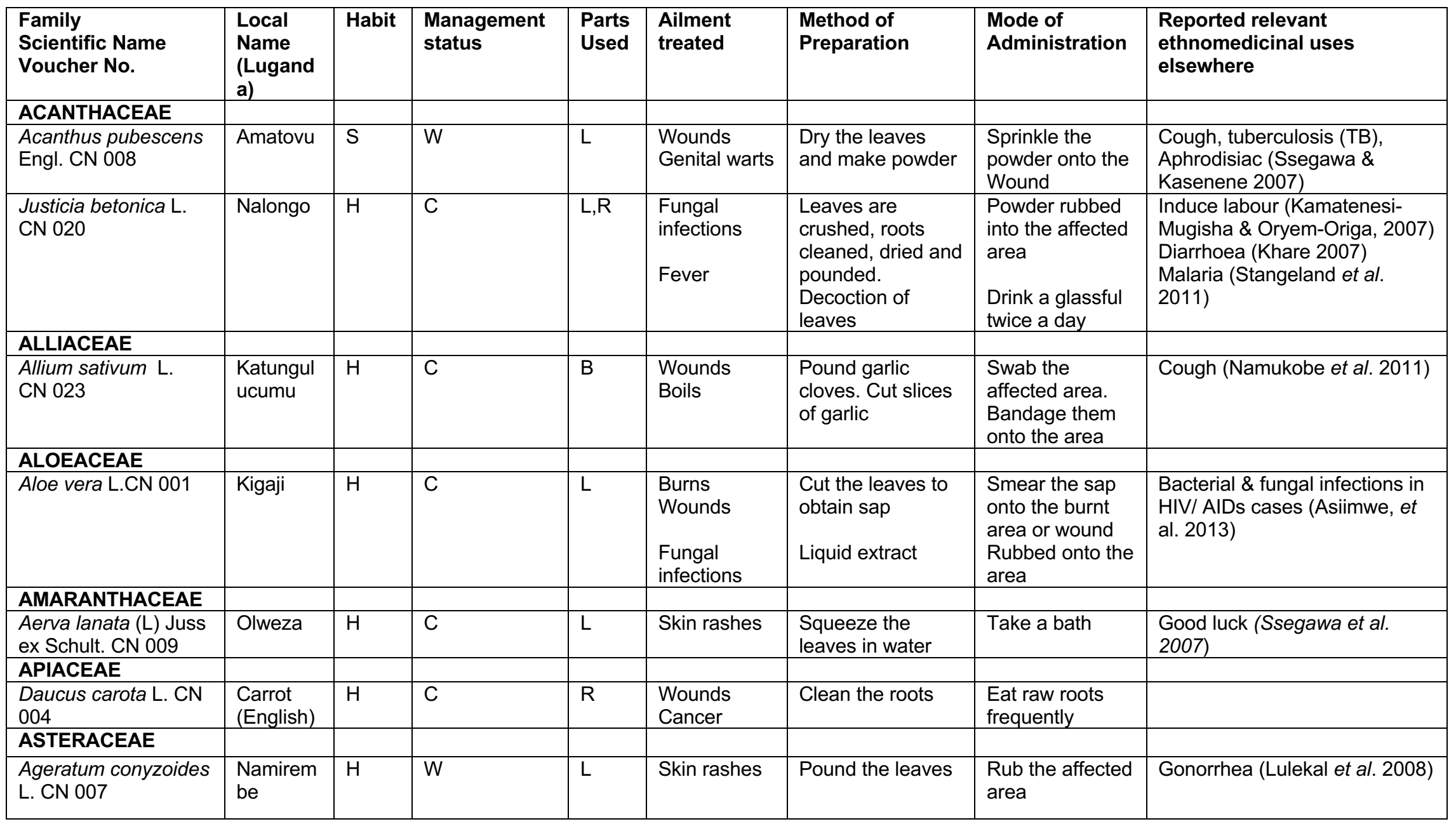

Published: 23 June 2019

http://dx.doi.org/10.32859/era.18.20.1-17 
Ethnobotany Research and Applications

\begin{tabular}{|c|c|c|c|c|c|c|c|c|}
\hline $\begin{array}{l}\text { Aspilia africana C.D } \\
\text { Adams (Pers.) CN } \\
015\end{array}$ & Makayi & $\mathrm{H}$ & W & $\mathrm{Ar}$ & Ringworm & $\begin{array}{l}\text { Pound the aerial } \\
\text { parts }\end{array}$ & $\begin{array}{l}\text { Rub the infected } \\
\text { area }\end{array}$ & $\begin{array}{l}\text { Haemorrhoids (Soladoye et al. } \\
\text { 2010) }\end{array}$ \\
\hline $\begin{array}{l}\text { Bidens pilosa } \mathrm{L} . \\
\text { CN002 }\end{array}$ & Ssere & $\mathrm{H}$ & W & L & Wounds & $\begin{array}{l}\text { Pound the fresh } \\
\text { leaves }\end{array}$ & $\begin{array}{l}\text { Apply the liquid } \\
\text { extract onto the } \\
\text { wound to effect } \\
\text { healing and } \\
\text { quick clotting }\end{array}$ & $\begin{array}{l}\text { Eye infection, nose bleeding, } \\
\text { yellow fever, diarrhoea, ulcers } \\
\text { (Namukobe et al. 2011) } \\
\text { Wound (Kamatenesi et al. } \\
\text { 2011, Ssegawa et al. 2007) }\end{array}$ \\
\hline $\begin{array}{l}\text { Conyza floribunda } \\
\text { Kunth. CN } 003\end{array}$ & Kafumbe & $\mathrm{H}$ & W & L & Ringworm & Crush the leaves & $\begin{array}{l}\text { Rub the infected } \\
\text { area }\end{array}$ & $\begin{array}{l}\text { Colic pain, Stomach ache, } \\
\text { syphilis, fever (Asiimwe et al. } \\
\text { 2014) }\end{array}$ \\
\hline $\begin{array}{l}\text { Erlangea tomentosa } \\
\text { S.Moore (Oliv \& } \\
\text { Hiern) CN } 012\end{array}$ & Ettwatwa & $\mathrm{H}$ & W & $\mathrm{L}$ & $\begin{array}{l}\text { Promotes a } \\
\text { healthy skin }\end{array}$ & $\begin{array}{l}\text { Squeeze leaves in } \\
\text { water }\end{array}$ & Take a bath & $\begin{array}{l}\text { Indigestion (Ssegawa etal. } \\
2007 \text { ) }\end{array}$ \\
\hline $\begin{array}{l}\text { Vernonia auriculifera } \\
\text { Hern. CN } 011\end{array}$ & $\begin{array}{l}\text { Kikokoo } \\
\text { ma }\end{array}$ & $S$ & W & $\mathrm{L}$ & $\begin{array}{l}\text { Fights skin } \\
\text { infections }\end{array}$ & $\begin{array}{l}\text { Squeeze leaves in } \\
\text { water }\end{array}$ & $\begin{array}{l}\text { Take a bath } \\
\text { occasionally }\end{array}$ & \\
\hline $\begin{array}{l}\text { Vernonia cinerea (L.) } \\
\text { Less. CN } 010\end{array}$ & $\begin{array}{l}\text { Kayayan } \\
\text { a }\end{array}$ & Sh & W & $\mathrm{L}$ & $\begin{array}{l}\text { Skin } \\
\text { infections }\end{array}$ & $\begin{array}{l}\text { Pound the fresh } \\
\text { leaves }\end{array}$ & $\begin{array}{l}\text { Rub the infected } \\
\text { area }\end{array}$ & $\begin{array}{l}\text { Good luck (Ssegawa et al. } \\
\text { 2007) }\end{array}$ \\
\hline $\begin{array}{l}\text { Spathodea } \\
\text { campanulata P. Beav. } \\
\text { CN } 005\end{array}$ & $\begin{array}{l}\text { Kifabaka } \\
\text { zi }\end{array}$ & $\mathrm{T}$ & W & L,B & $\begin{array}{l}\text { Skin rash } \\
\text { Genital warts }\end{array}$ & $\begin{array}{l}\text { Decoct the leaves } \\
\text { and bark. Add } \\
\text { some salt }\end{array}$ & $\begin{array}{l}\text { Drink regularly } \\
\text { Compress the } \\
\text { affected area }\end{array}$ & TB (Bunalema et al. 2014) \\
\hline \multicolumn{9}{|l|}{ BRASSICACEAE } \\
\hline $\begin{array}{l}\text { Brassica oleracea L. } \\
\text { CN } 013\end{array}$ & Cabbage & $\mathrm{H}$ & $\mathrm{C}$ & $\mathrm{L}$ & Wounds & Pound the leaves & $\begin{array}{l}\text { Put sap onto the } \\
\text { wound }\end{array}$ & \\
\hline $\begin{array}{l}\text { Cardamine } \\
\text { trichocarpa Hochst. } \\
\text { ex.A. Rich. CN } 014\end{array}$ & $\begin{array}{l}\text { Magereg } \\
\text { ankoko }\end{array}$ & $\mathrm{H}$ & W & $\mathrm{L}$ & $\begin{array}{l}\text { Wounds } \\
\text { Boils }\end{array}$ & Pound the leaves & $\begin{array}{l}\text { Apply } \\
\text { Compress }\end{array}$ & \\
\hline \multicolumn{9}{|l|}{ BURSERACEAE } \\
\hline $\begin{array}{l}\text { Canarium } \\
\text { schweinfurthii Engl. } \\
\text { CN } 006\end{array}$ & Omuwafu & $\mathrm{H}$ & C & SP & Scabies & $\begin{array}{l}\text { Cut the bark to get } \\
\text { the viscous resin }\end{array}$ & $\begin{array}{l}\text { Rub the affected } \\
\text { area. Applied as } \\
\text { ointment }\end{array}$ & $\begin{array}{l}\text { Possessed by spirits (Ssegawa } \\
\text { et al. 2007) }\end{array}$ \\
\hline CAPPARACEAE & & & & & & & & \\
\hline
\end{tabular}

Published: 23 June 2019

http://dx.doi.org/10.32859/era.18.20.1-17 
Ethnobotany Research and Applications

\begin{tabular}{|c|c|c|c|c|c|c|c|c|}
\hline $\begin{array}{l}\text { Cleome gynandra L. } \\
\text { CN } 019\end{array}$ & Ejobyo & $\mathrm{H}$ & $\mathrm{C}, \mathrm{W}$ & $\mathrm{F}$ & $\begin{array}{l}\text { Fungal } \\
\text { infections }\end{array}$ & $\begin{array}{l}\text { Squeeze the } \\
\text { flowers }\end{array}$ & $\begin{array}{l}\text { Rub the affected } \\
\text { area }\end{array}$ & $\begin{array}{l}\text { Headache, ringworms, eye } \\
\text { disease (Kamatenesi et al. } \\
\text { 2011) }\end{array}$ \\
\hline \multicolumn{9}{|l|}{ CARICACEAE } \\
\hline $\begin{array}{l}\text { Carica papaya L. CN } \\
018\end{array}$ & Epapari & $\mathrm{H}$ & $\mathrm{C}$ & $\mathrm{Fr}$ & $\begin{array}{l}\begin{array}{l}\text { Fungal } \\
\text { infections }\end{array} \\
\text { Burns } \\
\begin{array}{l}\text { To soften the } \\
\text { skin }\end{array}\end{array}$ & $\begin{array}{l}\text { Cut the unripe fruit } \\
\text { to extract the latex } \\
\text { A few drops of the } \\
\text { pawpaw sap is } \\
\text { boiled in water } \\
\text { Cut the unripe fruit } \\
\text { into pieces. Boil } \\
\text { the cut pieces } \\
\text { Mix the resultant } \\
\text { liquid with } \\
\text { Vaseline }\end{array}$ & $\begin{array}{l}\text { Rub the affected } \\
\text { area with the } \\
\text { latex } \\
\text { Use the } \\
\text { resulting water } \\
\text { to wash the burn } \\
\text { Smear body } \\
\text { parts especially, } \\
\text { hands } \\
\text { Apply the sap to } \\
\text { wounds }\end{array}$ & $\begin{array}{l}\text { Anaemia (Lulekal et al. 2008), } \\
\text { Malaria Stangeland et al. } \\
\text { 2011) }\end{array}$ \\
\hline \multicolumn{9}{|l|}{ CLUSIACEAE } \\
\hline $\begin{array}{l}\text { Psorosperrnum } \\
\text { febrifugum Sprach } \\
\text { CN } 021\end{array}$ & $\begin{array}{l}\text { Akanziro } \\
\text { nziro }\end{array}$ & $\mathrm{T}$ & W & $B$ & Skin rashes & $\begin{array}{l}\text { Dry the bark. } \\
\text { Pound into } \\
\text { powder. Mix the } \\
\text { powder with } \\
\text { Vaseline }\end{array}$ & $\begin{array}{l}\text { Smear all over } \\
\text { the skin }\end{array}$ & $\begin{array}{l}\text { Syphilis, skin rash, induction of } \\
\text { labour, pre-hepatic jaundice, } \\
\text { fever (Ssegawa \& Kasenene } \\
\text { 2007) }\end{array}$ \\
\hline \multicolumn{9}{|l|}{ CRASSULACEAE } \\
\hline $\begin{array}{l}\text { Kalanchoe pinnata L. } \\
\text { CN } 022\end{array}$ & Ekiyondo & $\mathrm{H}$ & W,C & $\mathrm{L}$ & $\begin{array}{l}\text { Wounds } \\
\text { Burns }\end{array}$ & $\begin{array}{l}\text { Pound } 1 \text { handful } \\
\text { of washed leaves, } \\
\text { add } 1 \text { teaspoon of } \\
\text { sugar, heat up the } \\
\text { mass until it turns } \\
\text { brown } \\
\text { Pounded leaves } \\
\text { are boiled for a } \\
\text { short time in a } \\
\text { little water }\end{array}$ & $\begin{array}{l}\text { Use as a wound } \\
\text { dressing } \\
\text { Apply as a } \\
\text { poultice }\end{array}$ & $\begin{array}{l}\text { Diarrhoea, immune boosting } \\
\text { (Nalumansi et al. 2014) }\end{array}$ \\
\hline \multicolumn{9}{|l|}{ CURCUBITACEAE } \\
\hline $\begin{array}{l}\text { Cucumis sativus L. } \\
\text { CN } 052\end{array}$ & Akatanga & $\mathrm{H}$ & W & L & $\begin{array}{l}\text { Skin } \\
\text { blemishes }\end{array}$ & $\begin{array}{l}\text { Squeeze the } \\
\text { leaves in water }\end{array}$ & Take a bath & \\
\hline $\begin{array}{l}\text { Mormodica foetida } \\
\text { K.Schum CN } 029\end{array}$ & Ebombo & $\mathrm{H}$ & W & L & $\begin{array}{l}\text { Body odours } \\
\text { Itching skin }\end{array}$ & $\begin{array}{l}\text { Squeeze the } \\
\text { leaves in water }\end{array}$ & $\begin{array}{l}\text { Bathe the whole } \\
\text { body }\end{array}$ & $\begin{array}{l}\text { Diarrhoea, Syphilis, } \\
\text { gonorrhoea (Okello \& } \\
\text { Ssegawa, 2007) }\end{array}$ \\
\hline
\end{tabular}

Published: 23 June 2019

http://dx.doi.org/10.32859/era.18.20.1-17 
Ethnobotany Research and Applications

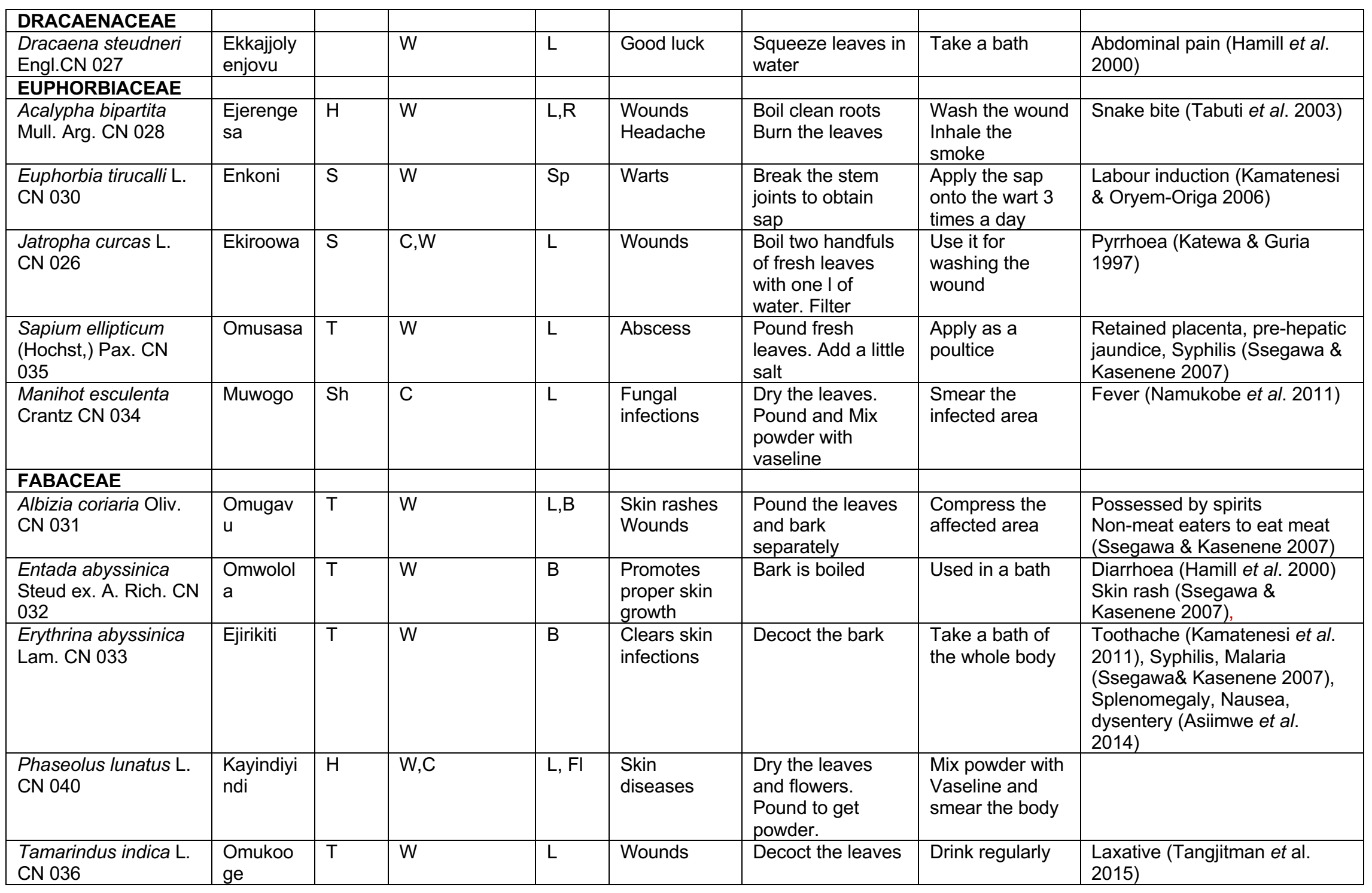

Published: 23 June 2019

http://dx.doi.org/10.32859/era.18.20.1-17 
Ethnobotany Research and Applications

\begin{tabular}{|c|c|c|c|c|c|c|c|c|}
\hline \multicolumn{9}{|l|}{ LAMIACEAE } \\
\hline $\begin{array}{l}\text { Hoslundia opposita } \\
\text { Vahl. CN } 037\end{array}$ & Kamunye & Sh & W & L & $\begin{array}{l}\text { Wounds } \\
\text { Toothache }\end{array}$ & $\begin{array}{l}\text { Squeeze leaves } \\
\text { Squeeze a } \\
\text { handful in quarter } \\
\text { litre of boiled } \\
\text { water. Add some } \\
\text { salt }\end{array}$ & $\begin{array}{l}\text { Compress the } \\
\text { wound } \\
\text { Use as a garble }\end{array}$ & $\begin{array}{l}\text { Epilepsy (Kamatenesi et al. } \\
\text { 2011) } \\
\text { Malaria (Stangeland et al. } \\
\text { 2011) }\end{array}$ \\
\hline $\begin{array}{l}\text { Leonotis nepetifolia } \\
\text { (L.) R. Br. CN } 038\end{array}$ & $\begin{array}{l}\text { Kifumufu } \\
\text { mu }\end{array}$ & $\mathrm{H}$ & W & $\mathrm{L}, \mathrm{FI}$ & Wounds & $\begin{array}{l}\text { Dry leaves. Make } \\
\text { into powder }\end{array}$ & $\begin{array}{l}\text { Sprinkle powder } \\
\text { to the wound to } \\
\text { effect quick } \\
\text { healing }\end{array}$ & $\begin{array}{l}\text { Backache (Ssegawa \& } \\
\text { Kasenene 2007) }\end{array}$ \\
\hline $\begin{array}{l}\text { Plectranthus barbatus } \\
\text { Andr. CN } 039\end{array}$ & $\begin{array}{l}\text { Kibwank } \\
\text { ulata }\end{array}$ & $\mathrm{H}$ & W,C & L & $\begin{array}{l}\text { Wounds } \\
\text { Ulcers }\end{array}$ & $\begin{array}{l}\text { Squeeze the } \\
\text { leaves. Dry leaves } \\
\text { under shade for } \\
\text { four days. Then } \\
\text { dry under direct } \\
\text { sunlight for a few } \\
\text { hours and pound } \\
\text { Squeeze the } \\
\text { leaves } \\
\text { Make a decoction }\end{array}$ & $\begin{array}{l}\text { Apply the liquid } \\
\text { extract to the } \\
\text { wounds till it } \\
\text { heals } \\
\text { Mix with } \\
\text { Vaseline and } \\
\text { smear } \\
\text { Drink regularly } \\
\end{array}$ & $\begin{array}{l}\text { TB (Bunalema et al. 2014), } \\
\text { Deworming, sinuses, Urinary } \\
\text { tract infections, Skin allergy, } \\
\text { stomach infections, syphilis } \\
\text { (Asiimwe et al. 2014) }\end{array}$ \\
\hline \multicolumn{9}{|l|}{ LAURACEAE } \\
\hline $\begin{array}{l}\text { Persea americana } \\
\text { Mill. } \\
\text { CN } 042\end{array}$ & Vakedo & $\mathrm{T}$ & $\mathrm{C}, \mathrm{W}$ & $\mathrm{Fr}$ & $\begin{array}{l}\text { Nourishes } \\
\text { the skin }\end{array}$ & $\begin{array}{l}\text { Cut up the ripe } \\
\text { fruit }\end{array}$ & $\begin{array}{l}\text { A dressing of } \\
\text { the avocado } \\
\text { flesh works as a } \\
\text { natural emulsion } \\
\text { and nourishes } \\
\text { infected or dry } \\
\text { skin }\end{array}$ & $\begin{array}{l}\text { Immune booster (Asiimwe et } \\
\text { al. 2013), Malaria, Diarrhoea, } \\
\text { vomiting (Nalumansi et al. } \\
2014 \text { ) }\end{array}$ \\
\hline \multicolumn{9}{|l|}{ MORACEAE } \\
\hline $\begin{array}{l}\text { Ficus ovata Vahl CN } \\
041\end{array}$ & $\begin{array}{l}\text { Omukoko } \\
\text { owe }\end{array}$ & $\mathrm{T}$ & $\mathrm{W}$ & Sp & Warts & $\begin{array}{l}\text { Detach fresh } \\
\text { leaves from the } \\
\text { stem to get the } \\
\text { white sap }\end{array}$ & $\begin{array}{l}\text { Apply the sap } \\
\text { onto the wart till } \\
\text { it breaks off the } \\
\text { skin }\end{array}$ & \\
\hline \multicolumn{9}{|l|}{ MORINGACEAE } \\
\hline $\begin{array}{l}\text { Moringa oleifera Lam } \\
\mathrm{CN} 043\end{array}$ & Mulinga & $\mathrm{T}$ & $\mathrm{C}$ & $\begin{array}{l}\text { Se, L } \\
F, R\end{array}$ & Wounds, & $\begin{array}{l}\text { Pound the fresh } \\
\text { leaves. Dry fresh } \\
\text { leaves and pound. }\end{array}$ & $\begin{array}{l}\text { Smear the } \\
\text { wound. Powder } \\
\text { is added to }\end{array}$ & $\begin{array}{l}\text { Malaria (Ssegawa \& Kasenene } \\
\text { 2007) }\end{array}$ \\
\hline
\end{tabular}

Published: 23 June 2019

http://dx.doi.org/10.32859/era.18.20.1-17 


\begin{tabular}{|c|c|c|c|c|c|c|c|c|}
\hline & & & & & $\begin{array}{l}\text { Fungal } \\
\text { infections } \\
\text { Boosts good } \\
\text { health }\end{array}$ & $\begin{array}{l}\text { A paste is made } \\
\text { from seeds } \\
\text { Boil the roots } \\
\text { Powder is } \\
\text { obtained from } \\
\text { flowers. } \\
\text { Fresh leaves are } \\
\text { prepared as } \\
\text { vegetable }\end{array}$ & $\begin{array}{l}\text { Vaseline and } \\
\text { smeared to clear } \\
\text { the skin. } \\
\text { Drink regularly. } \\
\text { Taken as tea }\end{array}$ & $\begin{array}{l}\text { Haemorrhoids (Soladoye et al. } \\
\text { 2010), TB (Bunalema et al. } \\
\text { 2014) }\end{array}$ \\
\hline \multicolumn{9}{|l|}{ MUSACEAE } \\
\hline $\begin{array}{l}\text { Ensete ventricosum ( } \\
\text { Welw) Cheesman } \\
\text { CN } 044\end{array}$ & Ekitembe & $\mathrm{H}$ & W & $\mathrm{Ps}, \mathrm{Fr}$ & Skin ulcers & $\begin{array}{l}\text { Boil the pseudo } \\
\text { stem thoroughly }\end{array}$ & Drink frequently & $\begin{array}{l}\text { Expel placentas, aphrodisiac, } \\
\text { Asthenia (for women) (Hamill } \\
\text { et al. 2000) }\end{array}$ \\
\hline \multicolumn{9}{|l|}{ MYRTACEAE } \\
\hline $\begin{array}{l}\text { Maesopsis eminii } \\
\text { Engl. CN } 050\end{array}$ & Musizi & $\mathrm{T}$ & W & $B$ & $\begin{array}{l}\text { Boosts skin } \\
\text { growth }\end{array}$ & Boil the bark & Take a bath & \\
\hline \multicolumn{9}{|l|}{ PHYLLANTHACEAE } \\
\hline $\begin{array}{l}\text { Bridelia micrantha } \\
\text { (Hochst) Baill. CN } \\
049\end{array}$ & $\begin{array}{l}\text { Katazami } \\
\mathrm{t}\end{array}$ & $\mathrm{S}$ & W & $\mathrm{L}$ & $\begin{array}{l}\text { Skin } \\
\text { infections } \\
\text { Rashes }\end{array}$ & $\begin{array}{l}\text { Dry the leaves. } \\
\text { Pound into } \\
\text { powder }\end{array}$ & $\begin{array}{l}\text { Rub powder into } \\
\text { the rash }\end{array}$ & $\begin{array}{l}\text { Tapeworm, Abdominal pain } \\
\text { (Hamill et al. 2000) }\end{array}$ \\
\hline \multicolumn{9}{|l|}{ PHYTOLACACEAE } \\
\hline $\begin{array}{l}\text { Phytolacca } \\
\text { dodecandra L' Hĕr } \\
\text { CN } 045\end{array}$ & Oluwoko & $\mathrm{H}$ & W & $\begin{array}{l}\text { Se, L, } \\
\mathrm{R}\end{array}$ & $\begin{array}{l}\text { Burns } \\
\text { Wounds }\end{array}$ & $\begin{array}{l}\text { Squeeze ripe } \\
\text { seeds. } \\
\text { Crush leaves to } \\
\text { get a foam } \\
\text { Burn the leaves to } \\
\text { ash. } \\
\text { Dry the roots, } \\
\text { make powder }\end{array}$ & $\begin{array}{l}\text { Spread the } \\
\text { paste over the } \\
\text { burn. Topically } \\
\text { apply the foam } \\
\text { Sprinkle the ash } \\
\text { and root powder } \\
\text { onto the burn } \\
\text { and wound }\end{array}$ & $\begin{array}{l}\text { Relaxation of pelvic region for } \\
\text { child delivery (Ssegawa \& } \\
\text { Kasenene 2007) }\end{array}$ \\
\hline \multicolumn{9}{|l|}{ PIPERACEAE } \\
\hline $\begin{array}{l}\text { Piper guineense } \\
\text { Schumach. Thonn. } \\
\text { CN } 046\end{array}$ & $\begin{array}{l}\text { Kigaman } \\
\text { sole }\end{array}$ & $\mathrm{V}$ & W & $\mathrm{R}, \mathrm{L}$ & $\begin{array}{l}\text { Skin } \\
\text { infections } \\
\text { Venereal } \\
\text { diseases, } \\
\text { Cough } \\
\end{array}$ & $\begin{array}{l}\text { Decoct leaves. } \\
\text { Make a brew from } \\
\text { roots }\end{array}$ & $\begin{array}{l}\text { Take a bath. } \\
\text { Take orally }\end{array}$ & $\begin{array}{l}\text { Haemorrhoids (Soladoye et al. } \\
\text { 2010) }\end{array}$ \\
\hline
\end{tabular}

Published: 23 June 2019

http://dx.doi.org/10.32859/era.18.20.1-17 
Ethnobotany Research and Applications

\begin{tabular}{|c|c|c|c|c|c|c|c|c|}
\hline \multicolumn{9}{|l|}{ POACEAE } \\
\hline $\begin{array}{l}\text { Cymbopogon citratus } \\
\text { ( D.C) Stapf. CN } 047\end{array}$ & Ekisubi & $G$ & $\mathrm{C}, \mathrm{W}$ & L & Skin care & Distill the leaves & $\begin{array}{l}\text { Scented product } \\
\text { is added into } \\
\text { skin care } \\
\text { products }\end{array}$ & $\begin{array}{l}\text { Haemorrhoids (Soladoye et al. } \\
\text { 2010), Cough, TB (Asiimwe et } \\
\text { al. 2013) }\end{array}$ \\
\hline \multicolumn{9}{|l|}{ SOLANACEAE } \\
\hline $\begin{array}{l}\text { Nicotiana tabacum L. } \\
\text { CN } 048\end{array}$ & Taaba & $\mathrm{H}$ & W, C & $\mathrm{L}$ & Ringworm & $\begin{array}{l}\text { Harvest clean } \\
\text { insect free leaves }\end{array}$ & $\begin{array}{l}\text { Rub affected } \\
\text { areas with } \\
\text { leaves }\end{array}$ & $\begin{array}{l}\text { Haemorrhoids (Soladoye et al. } \\
\text { 2010) }\end{array}$ \\
\hline $\begin{array}{l}\text { Lycopersicon } \\
\text { esculentum Mill. } \\
\text { CN } 049\end{array}$ & Nyanya & $\mathrm{H}$ & C & $\mathrm{L}, \mathrm{Fr}$ & $\begin{array}{l}\text { Fungal } \\
\text { infections }\end{array}$ & $\begin{array}{l}\text { Pound the leaves, } \\
\text { harvest ripe fruits }\end{array}$ & $\begin{array}{l}\text { Rub affected } \\
\text { area } \\
\text { Eat as a } \\
\text { vegetable }\end{array}$ & $\begin{array}{l}\text { Headache (Ssegawa \& } \\
\text { Kasenene 2007) }\end{array}$ \\
\hline $\begin{array}{l}\text { Solanum nigrum L. } \\
\text { CN } 050\end{array}$ & Ensugga & $\mathrm{H}$ & W, C & $\mathrm{L}$ & $\begin{array}{l}\text { Nourishes } \\
\text { skin }\end{array}$ & $\begin{array}{l}\text { Harvest young } \\
\text { aerial parts, steam } \\
\text { them }\end{array}$ & $\begin{array}{l}\text { Eat as a } \\
\text { vegetable }\end{array}$ & \\
\hline \multicolumn{9}{|l|}{ ZINGIBERACEAE } \\
\hline $\begin{array}{l}\text { Aframomum } \\
\text { angustifolium K. } \\
\text { Schum. CN } 051\end{array}$ & $\begin{array}{l}\text { Matungul } \\
\mathrm{u}\end{array}$ & $\mathrm{H}$ & W & $\mathrm{Fr}, \mathrm{R}$ & De-wormer & $\begin{array}{l}\text { Fruits and roots } \\
\text { are washed }\end{array}$ & $\begin{array}{l}\text { Eaten as a } \\
\text { snack }\end{array}$ & $\begin{array}{l}\text { Dysentery (Gazzaneo et al. } \\
\text { 2005) }\end{array}$ \\
\hline
\end{tabular}

Parts used: Ar - Aerial parts; B - Bark; FI - Flower; Fr - Fruit; Se - Seed; Sp -Sap; R - Root, Ps- pseudo stem

Growth form: G - Grass; H - Herb; Sh - Shrub; T - Tree; V - Vine.

Management status: C - Cultivated; W - Wild. 
Table 2. Percentage of plant species used to treat different skin disease categories

\begin{tabular}{|l|l|}
\hline $\begin{array}{l}\text { Skin disease } \\
\text { category }\end{array}$ & $\begin{array}{l}\text { Percentage } \\
\text { of plants } \\
\text { used for } \\
\text { treatment } \\
(\mathbf{N}=\mathbf{5 0})\end{array}$ \\
\hline Fungal infections & 36 \\
\hline Wounds & 32 \\
\hline Skin rash & 22 \\
\hline Warts & 10 \\
\hline Burns & 8 \\
\hline Boils & 6 \\
\hline Scabies & 2 \\
\hline
\end{tabular}

One plant could be used to treat more than one skin disease types

The commonest growth forms were herbs (55\%) followed by trees $(25 \%)$, shrubs $(17 \%)$ and vines $(1 \%)$ and grasses $(1 \%)$. The predominant use of herbs is attributed to their abundance all year round as reported in other studies from Uganda (Namukobe et al., 2011, Tugume et al., 2016). Herbs have also been found to contain high pharmacologically active ingredients compared to woody plants (Kamatenesi et al. 2011), a characteristic that makes them very useful for the management of ailments. $62 \%$ of medicinal plant species recorded were harvested from the wild. Cultivated plants constituted $21 \%$ while partially domesticated constituted $17 \%$. This indicates heavy dependence on wild sources instead of home gardens for medicinal plants collection and that cultivation of medicinal plants is not well embraced in the area. The high proportion of medicinal plant species from the wild exposes them to the risk of being endangered due to rampant clearance of wild habitats in favor of agriculture and human settlement. The low numbers of medicinal plant species cultivated could be attributed to low growth rates (Vines 2004). Being an urban area, land availability limits cultivation of medicinal plants. Sometimes cultivated plants are considered to be qualitatively inferior in comparison to wild plants (Schippmann et al. 2002). Cultivated plants included Moringa oleifera and Aloe vera which are mainly grown for commercial purposes. Medicinal plants under ex-situ conservation were grown under the mono-cropping system. This calls for an urgent need to document local flora ecosystems and growth requirements so that commonly used wild plants with a potential of being overexploited are conserved exsitu. Some of the cultivated plants such as Carica papaya, Manihot esculenta and Moringa oleifera are nutriceuticals, a factor that favors their cultivation. Dominant species identified according to gender of respondents were; Psorospermum febrifugum,
Albizia coriaria, Hoslundia opposita, Justicia betonica and Euphorbia tirucalli. More females used these plants for management of skin diseases compared to men (Fig. 2).

Leaves were the most commonly used parts (54\%), followed by roots and flowers $(11 \%)$ each while the other plant parts had proportions below $10 \%$. Leaves and roots generally form the most frequently used plant parts in traditional medicine as reported by Giday (2003) and Wondimu et al. (2007). Leaves are available all year round. Seeds, flowers and fruits are least used because they are seasonal. The bark and roots though moderately used require proper harvesting skills to avoid damaging the plants leading to their eventual death. The use of individual plant parts to treat skin diseases contrasts findings of Rahmatullah et al. (2011) in Bangladesh where whole plants were commonly used. Herbaceous plants can be used as whole plants because of the ease of extraction compared to trees or shrubs that present difficulty in harvesting wholly.

The methods of preparation of herbal medicines with their respective proportions of use were compresses $(12 \%)$, poultices $(24 \%)$, drying and pounding $(16 \%)$, sap extraction from fleshy parts by cutting (14\%), decoctions $(28 \%)$, squeezing in cold water $(14 \%)$, chewing $(10 \%)$ and burning to inhale the smoke $(2 \%)$. More than one preparation method could be used to prepare remedies from the same plant depending on plant parts. Sap and other resinous liquid extracts were obtained by cutting fleshy plant parts like those of Aloe and Euphorbia. Boiling was done to extract active ingredients and to increase the shelf life of herbal remedies. The mode of administration of the remedy for skin diseases was by topical application which involved rubbing or smearing the affected parts. Baths were occasionally applied. Cleome gynandra, Entanda abyssinica, and Plectranthus barbatus which were identified were also used to treat ringworms, skin rashes and skin allergy respectively in other areas (Table 1). Forty one plant species (82\%) identified were used for treatment of other ailments in Uganda and other parts Africa (Table 1). Such widespread use of these plants by different societies in different countries to a certain extent could be attributed to their effectiveness against the diseases they are used to treat (Maroyi 2013).

\section{Informant consensus agreement $\left(F_{i c}\right)$}

The $F_{\text {ic }}$ highlights the plant species with a healing potential for a specific major skin disease (Table 3). $F_{\text {ic }}$ values close to zero indicate a low degree of agreement among respondents about the use of a plant species for treatment of a particular skin condition. Fungal infections scored the highest $F_{\text {ic }}$ (0.9). High $F_{\text {ic }}$ values indicate that a species is used 
by a large proportion of informants and there is exchange of information. Species that are effective have high $F_{\text {ic }}$ values (Cakilcoglou et al. 2011). The important species used against fungal infections were Justicia betonica, Aloe vera, Aspilia africana, Conyza floribunda, Cleome gynandra, Carica papaya, Manihot esculenta, Moringa oleifera, Nicotiana tabacum and Lycoperscon esculentum. The high $F_{i c}$ values for fungal infections indicate agreement among respondents on the different plant species used to manage them and the significance residents of the study area attach to them.Boils and scabies had $F_{i c}$ of zero since each respondent reported different species used for boils and only one reported the use of Canarium schweinfurthii against scabies.

Table 3. Consensus agreement about uses of medicinal plants for skin disease category

\begin{tabular}{|l|l|l|l|}
\hline $\begin{array}{l}\text { Skin } \\
\text { disease } \\
\text { category }\end{array}$ & Ntaxa & Nur & $\mathbf{F}_{\text {ic }}$ \\
\hline $\begin{array}{l}\text { Fungal } \\
\text { infections }\end{array}$ & 36 & 19 & 0.9 \\
\hline Wounds & 32 & 20 & 0.7 \\
\hline Burns & 8 & 6 & 0.4 \\
\hline Warts & 10 & 7 & 0.5 \\
\hline Skin rash & 22 & 12 & 0.9 \\
\hline Boils & 6 & 6 & 0 \\
\hline Scabies & 1 & 1 & 0 \\
\hline
\end{tabular}

Fidelity leves (FL) of dominantly used plant species For each of the five dominantly used plant species a fidelity level was calculated to quantify their importance in the treatment of a major skin condition. Justicia betonica had the highest fidelity level of $89 \%$ against fungal infections. All the five plant species had high fidelity levels of greater than $50 \%$ (Table 4). This indicates the outstanding preference of $\mathrm{J}$. betonica against fungal infections of the skin.

Table 4. Fidelity levels (FL) of dominantly used medicinal plant species

\begin{tabular}{|l|l|l|l|l|}
\hline Plant species & Therapeutic use & $\mathbf{I}_{\mathbf{p}}$ & $\mathbf{I}_{\mathbf{u}}$ & FL (\%) \\
\hline Psorospermum febrifugum & Skin rash & 20 & 30 & 67 \\
\hline Albizia coriaria & Wounds & 31 & 35 & 89 \\
\hline Hos/undia opposita & Wounds & 15 & 25 & 60 \\
\hline Justicia betonica & Fungal infections & 30 & 40 & 75 \\
\hline Euphorbia tirucalli & Warts & 10 & 15 & 67 \\
\hline
\end{tabular}

Other uses of medicinal plant species identified in the current study

Some of the identified medicinal plant species were reported for other uses by different studies. These were important for food, fodder, material culture, firewood, charcoal, ceremonial purposes and income generation (Table 5). The multipurpose use of these species makes them an important source of livelihood which makes them susceptible to overexploitation. Multipurpose species need to be sustainably used and conserved to avoid negative impacts on the livelihoods of the users and the environment.

\section{Conclusions}

The traditional knowledge about the use of medicinal plants for management of skin diseases was diverse in Katabi Sub-County. The dominant plant species used were; Psorospermum febrifugum, Albizia coriaria, Hos/undia opposita, Justicia betonica, and Euphorbia tirucalli.

The study showed that medicinal plants have a great potential to cure different skin diseases. Fungal infections were the most common skin diseases treated using medicinal plants and had the highest
$F_{i c}$ indicating a high level of agreement among respondents of the plant species used to treat them. The important species used against fungal infections were Justicia betonica, Aloe vera, Aspilia africana, Conyza floribunda, Cleome gynandra, Carica papaya, Manihot esculenta, Moringa oleifera, Nicotiana tabacum and Lycoperscon esculentum. Justicia betonica had the highest fidelity level and thus could be considered a potential plant for further pharmacological investigations that could lead to the discovery of new drugs against fungal skin infections. The dominantly used species deserve priority for ex-situ conservation so as to minimize dangers of overexploitation. Some of the medicinal plant species identified have other uses which puts them on high demand leading to overexploitation.

Table 5. Other ethnobotanical uses of some medicinal plant species identified

\begin{tabular}{|l|l|}
\hline Plant species & Ethnobotanical uses \\
\hline Aloe vera & $\begin{array}{l}\text { Pulp edible as a } \\
\text { confection, vegetable } \\
\text { and pickles (Mathur \& } \\
\text { Joshi 2013) }\end{array}$ \\
\hline Bidens pilosa & $\begin{array}{l}\text { Vegetable (Senbeta et } \\
\text { al. 2013) }\end{array}$ \\
\hline
\end{tabular}




\begin{tabular}{|c|c|}
\hline Vernonia auriculifera & $\begin{array}{l}\text { Construction of bee hives } \\
\text { (Senbeta et al. 2013) }\end{array}$ \\
\hline Carica papaya & Edible fruit (Tabuti 2012) \\
\hline Albizia coriaria & $\begin{array}{lr}\text { Firewood, } & \text { charcoal, } \\
\text { construction } & \text { (Tabuti } \\
2012)\end{array}$ \\
\hline Entanda abyssinica & $\begin{array}{l}\text { Honey (Senbet et al. } \\
\text { 2013) }\end{array}$ \\
\hline Tamarindus indica & $\begin{array}{l}\text { Fruit (food) } \begin{array}{l}\text { Leaves } \\
\text { (fodder),Stem }\end{array} \\
\text { material for roofing) ( } \\
\text { Asase \& Oteng-Yeboah } \\
\text { 2012) }\end{array}$ \\
\hline Persea americana & $\begin{array}{l}\text { Firewood (Nyamukuru et } \\
\text { al. 2015) }\end{array}$ \\
\hline Ficus ovata & $\begin{array}{l}\text { Bedding, death, religious } \\
\text { and wedding } \\
\text { rituals(Kakudidi 2004); } \\
\text { material for wood } \\
\text { furniture (Senbeta et al. } \\
2013) \text {; cosmetics, } \\
\text { hairdressing, fodder, } \\
\begin{array}{l}\text { vegetable (Mathur \& } \\
\text { Joshi 2013) }\end{array}\end{array}$ \\
\hline Maesopsis eminii & $\begin{array}{l}\text { Timber, firewood (Tabuti } \\
\text { 2012) }\end{array}$ \\
\hline Nicotiana tabacum & $\begin{array}{l}\text { Wedding present to the } \\
\text { elderly (Kakudidi 2004) }\end{array}$ \\
\hline
\end{tabular}

\section{Recommendations}

The commonly used plant species should be screened for their haemostyptic, disinfectant, analgesic and antibiotic properties in order to develop new drugs. The therapeutic recipes revealed by the study need to be evaluated through phytochemical and pharmacological investigations to discover their active compounds.

There is need to document traditional medicine practices to avoid indigenous knowledge erosion in case the elderly die due to breakdown of traditional societal structures of knowledge transmission. Therefore documentation of traditional knowledge in the use of plant species to treat different ailments needs to be carried out in different cultures to prevent erosion of such information that could be collected into a database for future research and potential drug development.

Women who heavily relied on using herbal medicine are already burdened with other household activities. Thus, there is need to increase male knowledge about traditional medicine through sensitization. Additionally on-farm conservation of medicinal plants should be encouraged to supplement wild collection. Links between traditional medical practitioners, researchers, conventional health practitioners and pharmaceutical companies should be promoted to enhance sharing of knowledge about the potential of medicinal plants for new drug development.

\section{Declarations \\ List of abbreviations \\ WHO: World Health Organisation}

Ethics approval and consent to participate:

All participants gave their prior informed consent before any study.

\section{Competing interests}

The authors declare that they have no competing interests.

\section{Availability of data: N/A}

Funding: N/A

\section{Author's contributions}

Cissy conceptualized the study, designed the methods, conducted the ethnobotanical survey, analysed the data and drafted the manuscript. Patience and Clement conceptualized the idea of this manuscript and participated in reviewing the manuscript. Maud reviewed the manuscript. All authors read and approved the final manuscript.

\section{Acknowledgements}

We extend our sincere thanks to the Community of Katabi Sub-County who provided us with the information required for this study. Special thanks go to the staff of Makerere University Herbarium who helped us in plant identification.

\section{Literature cited}

Ahumuza T, Kirimuhuzya C. 2011. Qualitative (phytochemical) analysis and antifungal activity of Pentas decora (De Wild) a plant used traditionally to treat skin fungal infections in Western Uganda. Research in Pharmaceutical Biotechnology 3(7): 7584.

Asase A, Oteng-Yeboah AA. 2012. Plants used in Wechiau community Hippopotamus Sacnctuary in Northern Ghana. Ethnobotany Research and Applications 10:605-618.

Asiimwe S, Kamatenesi-Mugisha M, Namutebi A, Borg-Karlsson A, Musiimenta P. 2013. Ethnobotanical study of nutri-medicinal plants used for the management of HIVIAIDS opportunistic ailments among the local communities of Western Uganda. Journal of Ethnophamarcology 150: 639648.

Asiimwe S, Namutebi A, Borg-Karlsson A, Kamatenesi-Mugisha M, Oryema-Origa H. 2014. Documentation and Consensus of Indigenous 
knowledge on medicinal plants used by the local communities in Western Uganda. Journal of Natural Products Plant Resources 4(1): 34-42.

Badoni A, Badoni K. 2001. Ethnobotanical inheretence, Garhwal, Himalaya: Nature, culture and society. Eds Kandari OP, Gusain OP (Trans media, Srinagar Garhwal, India.

Bhat GP, Surolia N. 2001. In vitro anti-malarial activity of extracts of three plants used in traditional medicine in India. American Journal of Tropical Medicine and Hygiene 64: 304-308.

Bunalema L, Obakiro S, Tabuti JRS, Waako P. 2014. Knowledge on plants used traditionally in the treatment of tuberclosis in Uganda. Journal of Ethnopharmacology 151: 999-1004.

Cakilcioglu U, Khatun SL, Turkoglu I, Haytad S. Ethnopharmacological survey of medicinal plants in Maden (Elazig-Turkey). Journal of Ethnopharmacology 2011;137(1):469-86.

Chen SL, Yu H, Luo HM, Wu Q, Li CF, Steinmetz A. 2016. Conservation and sustainable use of medicinal plants: problems, progress and prospects. Chinese Medicine 11(1):37.

Friedman J, Yaniv Z, Dafni A, Palewitch D. A preliminary classification of the healing potential of medicinal plants, based on rational analysis of an ethnopharmacological field survey among Bedouins in Negev Desert, Israel. Journal of Ethnopharmacology 1986;16:275-87.

Galabuzi C, Agea JG, Fungo BL, KAmoga RMN. 2010. Traditional medicine as an alternative from of healthcare system: Apreliminary case study of Nangabo Sub-County, Central Uganda. African Journal of Traditional Complemetary and Alternative Medicine 7(1):11-16.

Gazzaneo LRS, Lucena RFP, Albuquerque UP. 2005. "Knowledge and use of medicinal plants by local specialists in a region of Atlantic Forest in the state of Pernambuco (Northern Brazil). Journal of Ethnobiology and Ethnomedicine 1: 9.

Giday M, Asfaw Z, Elmqvist T, Woldu Z. 2003. An Ethnobotanica; study of medicinal plants used by the Zay people in Ethiopia. Journal of Ethnophamarcology 85: 43-52.

Gollin LX. 2004. Subtle and profound sensory attributes of medicinal plants among the Kenyah Leppo Ke of East Kilimantan, Borneo. Journal of Ethnobiology 24 (2):173-201.

Hamill FA, Apio S, Mubira NK, Mosango M, Bukenya-Ziraba, R, Maganyi OW, Soejarto DD.
2000. Traditional herbal drugs of Southern Uganda. . Journal of Ethnopharmacology 70: 281-300.

Iwu MM. 1993. Handbook of African medicinal plants. USA: CRC Press LLC.

Kakudidi EK. 2003. Cultural and social uses of plants from and around Kibale National Park, Western Uganda. African Journal of Ecology 42(Suppl.1):114-118.

Kala CP, Farooquee NA, Majila BS. 2005. Indigenous knowledge and medicinal plants used by Vaidyas in Uttarkhand, India. Natural Products Resources 4:195-204

Kamagaju L, Biziru E, Minari V, Morandiru R, Stevigny C, Ghanem G, Deuz P. 2013. An Ethnobotanical survey of medicinal plants used in Rwanda for voluntary depigmentation. Journal of Ethnopharmacology 150(2): 708-717.

Kamatenesi-Mugisha M, Oryem-Origa H. 2007. Medicinal Plants used to induce labour during child birth in Western uganda. Journal of Ethnopharmacology 109:1-9.

Kamatenesi MK, Acipa A, Oryema-Origa H. 2011. Medicinal plants of Otwal and Ngai sub counties in Oyam District, Northern Uganda. Journal of Ethnobiology and Ethnomedicine 7: 7.

Karuiki AC, Njoroge GN. 2011. Ethnobotanical and antimicrobial studies of some plants used in Kibwezi (Kenya) for management of lower respiratory tract infections. Africa Journal of Traditional, complementary and Alternative medicine 8(2): 144149.

Katende AB, Ssegawa P, Birnie A. 1999. Wild plant species and edible mushrooms of Uganda. SIDA Technical Handbook No.9. Nairobi.

Katewa SS, Guria BD. 1997. Ethnomedicinal observations on certain wild plants from Southern Aravalli hills in Rajasthan. Vadundhara 2: 85-88.

Khare CP. 2007. Indian Medicinal plants: An illustrated dictionary. Heidelberg: Springer-Verlag.

Kroeger A. 1983. Anthropological and socio-medical healthcare research in developing countries. Social Science and Medicine 17:147-161.

Lulekal E, Kelbessa E, Bekele T, Yinegar H. 2008. An Ethnobotanical study of medicinal plants in Mana Angetu District, Southern Ethiopia. Journal of Ethnobiology and Ethnomediciene 4: 10.

Mahbub M, Mazumder SH, Moshed MZ, Johan R, Hossan MS, Rahmatulla M. 2018. Folk medicinal use of plants to treat skin disorders in Chandpur District, 
Bangladesh. American Journal of Ethnomedicine 4(2):19.

Maroyi A. 2013. Traditional use of medicinal plants i South Central Zimbabwe: review \& perspectives. Journal of Ethnobiology and Ethnomedicine 9:31.

Mathur A, Joshi H. 2013. Ethnobotanical studies of the Tarai Region of Kauman, Uttarakhand, India. Ethnobotany Research and Applications 11:175203.

Morakinyo OM, Gree A, Oloruntoba EO. 2014. Prevalence of skin infections and hygiene practices among pupils in selected public primary schools in Ibadan, Nigeria. African Journal of Sustainable Development 4:2.

Musoke D, Boynton P, Butler C, Musoke MB. 2014. Health seeking behaviour and challenges in utilising health facilities in Wakiso District, Uganda. African Health Sciences 14(4):1046-1055.

Nalumansi P, Kamatenesi-Mugisha M, Anywar G. 2014. Medicinal plants used in Paediatric Health Care in Namungalwe Sub-County, Iganga District, Uganda. Nova Journal of Medical and Biological Sciences 2(3): 1-14.

Namukobe J, Kasenene JM, Kiremire BT, Byamukama R, Kamatenesi-Mugisha, M, Krief S, Dumontent V, Kabasa JD. 2011. Traditional plants used for medicinal purposes by local comminities around the Northern sector of Kibale National Park, Uganda. Journal of Ethnopharmacology 136: 236245.

Nyamukuru A, Nabatanzi A, Mpiira S, Tabuti JRS. 2015. Locally preferres woody species and their management in kiruhura and Arua districts, Uganda. Ethnobotany Research and Applications 14:049061.

Okello J, Ssegawa P. 2007. Plants used by communities of Ngai Sub-County, Apac District, Northern Uganda. African Journal of ecology, 45(1): 6-83.

Rahmatullah M, Jahan R, Azam FMS, Hossan S, Mollik MAH, Rahman T. 2011. Folk medicinal uses of verbanaceae family plants in Bangladesh. African Journal of Traditional Complementary and Alternative Medicine 8(S): 53-65.

Senbeta F, Gole TW, Denich M, Kellbesa E. 2013. Diversity of useful plants in the coffee forests of Ethiopia. Ethnoboatny Research and Applications 11:049-069.

Schippmann U, Leamann BJ, Cunningham AB. 2002. Impact of cultivation and Gathering of
Medicinal Plants on Biodiversity: Global trends and Issues. FAO, Rome.

Shaikh BT, Hatcher J. 2005. Health seeking behaviour and health service utilisation in Pakistan: challenging the policy makers. Journal of Public Health (Oxf) 1:49-54.

Soladoye MO, Adetayo MO, Chukwuma CE, Adetunji NA. 2010. Ethnobotanical survey of plants used in the treatment of Haemorrhoids in South Western Nigeria. Annals of biological research 73:175-185.

Ssegawa P, Kasenene JM. 2007. Medicinal plant diversity and uses in Sango bay area, Southern Uganda. Journal of Ethnopharmacology 113: 521540.

Stangeland T, Alele PE, Katuura E, Lye KA. 2011. Plants used to treat malaria in Nyakayojo SubCounty, Western Uganda. Journal of Ethnopharmacology 137: 154-166.

Tabassum N, Hamdani M. 2014. Plants used to treat skin diseases. Pharmacognosy Review; 8: 52-60.

Tabuti JRS, Dhillion SS, Lye KA. 2003. Traditional medicine in Bulamogi conty, Uganda: Its practitioners, users and viability. Journal of Ethnopharmacology 85: 119-129.

Tabuti JRS. 2012. Important woody plant species, their management and conservation status in Balawoli Subcounty, Uganda. Etnnobotany Research and Applications 10:605-618.

Tangjitman K, Wongsawad C, Kamwong K, Sukicho T, Trisonthi C. 2015. Ethnomedicnal plants used for digestive system disorders by the Karen of Northern Thailand. Journal of Ethnobiology and Ethnomedicine 11: 27.

Thomas E, Vandebroek K, Sanca S, Van Damme P. 2009. Cultural significance of medicinal plant families and species among Quechua farmers Apillapampa, Bolivia. Journal of Ethnopharmacology 122: 60-67.

Trotter RJ, Logan MH. Informant consensus. A new approach for identifying potentially effective medicinal plants. In: Etkin NL, editor. Plants in indigenous medicine and diet. Bedford Hills: Newyork: Redgrave; 1986. p. 91-112.

Tugume P, Kakudidi EK, Buyinza M, Namaalwa J, Kamatenesi M, Mucunguzi, P, Kalema J. 2016. Ethnobotanical survey of medicinal plant species used by communities around Mabira Central Forest Reserve, Uganda. Journal of Ethnobiology and Ethnomedicine 12: 5. 
Uslu H, Uyanik M, Ayyildiz A. 2008. Mycological examination of barber's tools about some fungal infections. Mycoses 5(5):447-450.

Vikram NS, MAikhuri RK, Vashishtha DP. 2011. Traditional healthcare practices among villages of Rawain Valley, Uttarkashi Uttarakhand, India. Indian Journal of Traditional Knowledge 10(3):533-537.

Vines G. 2004. Herbal harvests with a future towards sustainable sources for medicinal plants. Plantlife international, Salisbury.
Wondimu T, Asfaw Z, Kelbesa E. 2007. Ethnobotanical study of medicinal plants around Dheera town Arsi Zone. Ethiopia Journal of Ethnophamacology, 112: 152-161.

WHO 2002. Traditional medicine strategy 2002 2005. Geneva.

WHO 2005. Epidemiology and management of common skin diseases in children in developing countries. Department of child and adolscent Health and Development. WHO/FCHI/CA/05.12 